\title{
An efficient boundary element modeling of the time domain integral equations for thin wires radiating in a presence of a lossy media
}

\author{
D. Poljak, S. Antonijevic \& V. Doric \\ University of Split, Croatia
}

\begin{abstract}
The paper deals with the space-time Galerkin-Bubnov scheme of the Indirect Boundary Element Method for the solution of time domain integral equations for thin wires in the presence of lossy media of the Pocklington and Hallen type, respectively. The presence of a dissipative half-space is taken into account via the corresponding space-time reflection coefficients. Some illustrative computational examples related to the overhead wires and grounding electrodes are presented.

Keywords: boundary elements, time domain modeling, Hallen equation, Pocklington equation, thin wires.
\end{abstract}

\section{Introduction}

Generally, a direct time-domain analysis of thin wire configurations in the presence of lossy media can be carried out by using the appropriate space-time integral equations of either Pocklington or Hallen type [1-4]. One of the most efficient numerical solution approaches to both equation types is related to the Galerkin-Bubnov Indirect Boundary Element Method (GB-IBEM) [2]. When applied to the solution of the Hallen integral equation the method appears to be relatively complex comparing to various procedures for the solution of Pocklington equations, but, at the same time, it is proven to be highly efficient and accurate and unconditionally stable [2, 4]. On the other hand, the implementation of GB-IBEM to the solution of the Pocklington type equation is relatively simple, but suffers from numerical instabilities. The origin of these instabilities is the existence of space-time differential operator [2]. The GBIBEM solution of Pocklington equation in free space for certain values of time 
domain integration parameters has been presented in [5], while the Hallen equation solution via GB-IBEM have been obtained for thin wire configurations in the presence of a dielectric half-space, e.g. in [6]. The original formulation has been developed for a single wire located horizontally above [2] and a below real ground, respectively [7]. In both cases, the influence of the finitely conducting ground has been taken into account via the corresponding reflection coefficient. Nevertheless, the numerical solution was mostly limited to the cases in which the finite conductivity of the ground could be ignored. This approximation involves cases where the wires are sufficiently far from the two-media interface, or where the ground conductivity is appreciably low or very high, i.e. where the approximation of pure dielectric medium or perfect ground is considered. Through these approximations the time dependent part of the reflection coefficient function vanishes, and the resulting matrix equation simplifies significantly.

However, for the cases where these approximations are not valid, modifications to the original methods are required in order to include the ground conductivity [8]. The related reflection coefficient is space- time dependent, and the resulting convolution integrals have to be included in the matrix system and numerically computed. This leads to a significant increase in the overall computational cost of the method, and requires several modifications.

This paper compares the space-time Pocklington and Hallen equation approaches when the lossy ground effects are taken into account. The related GB-IBEM procedures for the solution of both equations, taking into account a finite value of the ground conductivity, are discussed. Some illustrative computational examples related to transient analysis of overhead wires and grounding electrodes are given in the paper, as well.

\section{Hallen integral equation formulation}

The straight thin wire of length $L$ and radius a located at height $h$ above a lossy ground is shown in Fig. 1.

The formulation is based on the space-time dependent integral equation of the Hallen type which does not contain a differential operator. The Hallen is therefore particularly useful for a numerical treatment, as this operator is the origin of numerical instabilities [2].

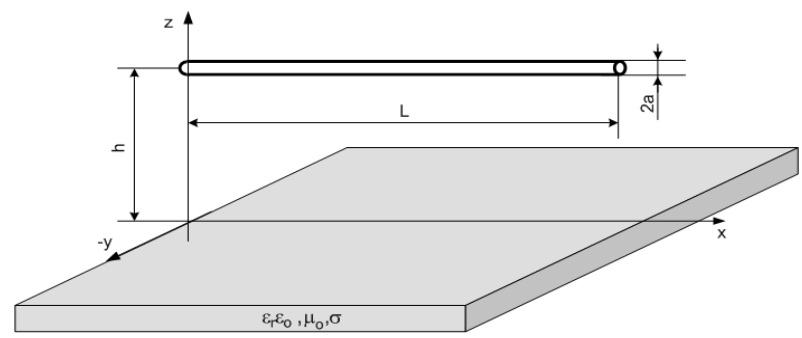

Figure 1: A straight thin wire above a real ground. 
The transient current $I(x, t)$ induced on the horizontal straight thin wire, depicted in Fig. 1, illuminated by a plane wave electric field can be assessed by solving the space-time Hallen integral equation:

$$
\begin{aligned}
& \int_{0}^{L} \frac{I\left(x^{\prime}, t-\frac{R}{c}\right)}{4 \pi R} d x^{\prime}-\int_{-\infty}^{t} \int_{0}^{L} r(\theta, \tau) \frac{I\left(x^{\prime}, t-\frac{R^{*}}{c}-\tau\right)}{4 \pi R^{*}} d x^{\prime} d \tau= \\
& \frac{1}{2 Z_{0}} \int_{0}^{L} E_{x}^{i n c}\left(x^{\prime}, t-\frac{\left|x-x^{\prime}\right|}{c}\right) d x^{\prime}+F_{0}\left(t-\frac{x}{c}\right)+F_{L}\left(t-\frac{L-x}{c}\right)
\end{aligned}
$$

where $R$ and $R^{*}$ are corresponding distances from the observation to the source point located on the real and image wire, respectively, $E_{x}^{e x c}$ is the tangential component of the excitation and $F_{0}, F_{L}$ are the unknown functions to account for the reflections at the wire free ends:

$$
\begin{aligned}
& F_{0}(t)=\sum_{n=0}^{\infty} K_{0}\left(t-\frac{2 n L}{c}\right)-\sum_{n=0}^{\infty} K_{L}\left(t-\frac{(2 n+1) L}{c}\right) \\
& F_{L}(t)=\sum_{n=0}^{\infty} K_{L}\left(t-\frac{2 n L}{c}\right)-\sum_{n=0}^{\infty} K_{0}\left(t-\frac{(2 n+1) L}{c}\right)
\end{aligned}
$$

defined in terms of auxiliary functions $K_{0}, K_{L}$ given by:

$$
\begin{aligned}
& K_{0}(t)=\int_{0}^{L} \frac{I\left(x^{\prime}, t-\frac{R_{0}}{c}\right)}{4 \pi R_{0}} d x^{\prime}-\int_{-\infty}^{t} \int_{0}^{L} r(\theta, \tau) \frac{I\left(x^{\prime}, t-\frac{R_{0}^{*}}{c}-\tau\right)}{4 \pi R_{0}^{*}} d x^{\prime} d \tau-\frac{1}{2 Z_{0}} \int_{0}^{L} E_{x}^{i n c}\left(x^{\prime}, t-\frac{x^{\prime}}{c}\right) d x^{\prime} \\
& K_{L}(t)=\int_{0}^{L} \frac{I\left(x^{\prime}, t-\frac{R_{L}}{c}\right)}{4 \pi R_{L}} d x^{\prime}-\int_{-\infty}^{t} \int_{0}^{L} r(\theta, \tau) \frac{I\left(x^{\prime}, t-\frac{R_{L}^{*}}{c}-\tau\right)}{4 \pi R_{L}^{*}} d x^{\prime} d \tau-\frac{1}{2 Z_{0}} \int_{0}^{L} E_{x}^{i n c}\left(x^{\prime}, t-\frac{L-x^{\prime}}{c}\right) d x^{\prime}
\end{aligned}
$$

where subscript 0 and $L$ is related to the distance from the observation point to the source point located on the real and image wire, respectively.

The ground effects are included in the formulation through the space-time dependent reflection coefficient for TM-polarization, [6] which, for convenience, can be written in the form:

$$
r(\theta, \tau)=r^{\prime}(\theta, \tau)+r^{\prime \prime}(\theta, \tau)
$$

where

$$
\begin{gathered}
r^{\prime}(\theta, t)=K \delta(t), r^{\prime \prime}(\theta, t)=\frac{4 \beta}{1-\beta^{2}} \frac{e^{-\alpha t}}{t} \sum_{n=1}^{\infty}(-1)^{n+1} n K^{n} I_{n}(\alpha t) \\
\tau=\frac{\sigma}{\varepsilon_{0} \varepsilon_{r}}, \quad \beta=\frac{\sqrt{\varepsilon_{r}-\sin ^{2} \theta}}{\varepsilon_{r} \cos \theta}, \gamma=\frac{\tau}{1-\frac{\sin ^{2} \theta}{\varepsilon_{r}}}, \quad \theta=\operatorname{arctg} \frac{\left|x-x^{\prime}\right|}{2 h}, \quad K=\frac{1-\beta}{1+\beta}, \quad \alpha=\frac{\tau}{2}
\end{gathered}
$$

Note that $I_{n}$ is the modified Bessel function of the first order, $n$-th degree. 
136 Boundary Elements and Other Mesh Reduction Methods XXXIII

\subsection{BEM procedure for Hallen equation}

Applying the weighted residual approach in the spatial domain and GB-IBEM procedure [2], the following local matrix system is obtained:

$$
\begin{aligned}
& {\left.[A]\{I\}_{i}\right|_{t-\frac{R}{c}}-\left.\left[A^{*}\right]\{I\}_{i}\right|_{t-\frac{R^{*}}{c}}-\left.\{\hat{A}\}\right|_{t-\frac{R^{*}}{c}}=\left.[B]\{E\}\right|_{t-\frac{\left|x-x^{*}\right|}{c}}} \\
& +\left.[C]\left\{\sum_{n=0}^{\infty} I^{n}\right\}_{i}\right|_{t-\frac{R_{0}}{c}-\frac{2 n L}{c}-\frac{x}{c}}-\left.\left[C^{*}\right]\left\{\sum_{n=0}^{\infty} I^{n}\right\}_{i}\right|_{t-\frac{R_{0}^{*}}{c}-\frac{2 n L}{c}-\frac{x}{c}}-\left.\left\{\sum_{n=0}^{\infty} \hat{C}^{n}\right\}\right|_{t-\frac{R_{0}^{*}}{c}-\frac{2 n L}{c}-\frac{x}{c}} \\
& -\left.[B]\left\{\sum_{n=0}^{\infty} E^{n}\right\}\right|_{t-\frac{x^{\prime}}{c}-\frac{2 n L}{c}-\frac{x}{c}}-\left.[D]\left\{\sum_{n=0}^{\infty} I^{n}\right\}_{i}\right|_{t-\frac{R_{L}}{c}-\frac{(2 n+1) L}{c}-\frac{x}{c}} \\
& +\left.\left[D^{*}\right]\left\{\sum_{n=0}^{\infty} I^{n}\right\}_{i}\right|_{t-\frac{R_{L}^{*}}{c}-\frac{(2 n+1) L}{c}-\frac{x}{c}}+\left.[B]\left\{\sum_{n=0}^{\infty} E^{n}\right\}\right|_{t-\frac{L-x^{\prime}}{c}-\frac{(2 n+1) L}{c}-\frac{x}{c}} \\
& +\left.\left\{\sum_{n=0}^{\infty} \hat{D}^{n}\right\}\right|_{t-\frac{R_{L}^{*}}{c}-\frac{(2 n+1) L}{c}-\frac{x}{c}}+\left.[D]\left\{\sum_{n=0}^{\infty} I^{n}\right\}_{i}\right|_{t-\frac{R_{L}}{c}-\frac{2 n L}{c}-\frac{L-x}{c}}-\left.\left[D^{*}\right]\left\{\sum_{n=0}^{\infty} I^{n}\right\}_{i}\right|_{t-\frac{R_{L}^{*}}{c}-\frac{2 n L}{c}-\frac{L-x}{c}} \\
& +\left.\left\{\sum_{n=0}^{\infty} \hat{D}^{n}\right\}\right|_{t-\frac{R_{L}^{*}}{c}-\frac{2 n L}{c}-\frac{L-x}{c}}-\left.[B]\left\{\sum_{n=0}^{\infty} E^{n}\right\}\right|_{t-\frac{L-x^{\prime}}{c}-\frac{2 n L}{c}-\frac{L-x}{c}}-\left.[C]\left\{\sum_{n=0}^{\infty} I^{n}\right\}_{i}\right|_{t-\frac{R_{0}}{c}-\frac{(2 n+1) L}{c}-\frac{L-x}{c}} \\
& +\left.\left.\left[C^{*}\right]\left\{\sum_{n=0}^{\infty} I^{n}\right\}\right|_{i}\right|_{t-\frac{R_{0}^{*}}{c}-\frac{(2 n+1) L}{c}-\frac{L-x}{c}}+\left.[B]\left\{\sum_{n=0}^{\infty} E^{n}\right\}\right|_{t-\frac{x^{\prime}}{c}-\frac{(2 n+1) L}{c}-\frac{L-x}{c}}-\left.\left\{\sum_{n=0}^{\infty} \hat{C}^{n}\right\}\right|_{t-\frac{R_{0}^{*}}{c}-\frac{(2 n+1) L}{c}-\frac{L-x}{c}}
\end{aligned}
$$

The space-dependent local matrices representing the interaction between $i$-th source and $j$-th observation element are defined, as follows:

$$
\begin{aligned}
& {[A]=\int_{\Delta \Delta_{j}} \int_{\Delta \Delta_{i}}\{f\}_{j}\{f\}_{i}^{T} \frac{1}{4 \pi R} d x^{\prime} d x ; \quad[B]=\frac{1}{2 Z_{0}} \int_{\Delta I_{j}} \int_{\Delta \Delta_{i}}\{f\}_{j}\{f\}_{i}^{T} d x^{\prime} d x} \\
& {[C]=\int_{\Delta l_{j}} \int_{\Delta \Delta_{i}}\{f\}_{j}\{f\}_{i}^{T} \frac{1}{4 \pi R_{0}} d x^{\prime} d x ; \quad[D]=\int_{\Delta_{l_{j}} \Delta_{j}}\{f\}_{j}\{f\}_{i}^{T} \frac{1}{4 \pi R_{L}} d x^{\prime} d x} \\
& {\left[A^{*}\right]=\int_{\Delta l_{j}} \int_{\Delta \Delta_{i}}\{f\}_{j}\{f\}_{i}^{T} \frac{r(\theta)}{4 \pi R^{*}} d x^{\prime} d x ;\left[C^{*}\right]=\int_{\Delta l_{j}} \int_{\Delta \Delta_{i}}\{f\}_{j}\{f\}_{i}^{T} \frac{r(\theta)}{4 \pi R_{0}^{*}} d x^{\prime} d x} \\
& {\left[D^{*}\right]=\int_{\Delta l_{j} \Delta \Delta_{i}}\{f\}_{j}\{f\}_{i}^{T} \frac{r(\theta)}{4 \pi R_{L}^{*}} d x^{\prime} d x ;}
\end{aligned}
$$

where $\{f\}$ stands for the shape functions, while additional time dependent vectors are given by: 


$$
\begin{aligned}
& \{\hat{A}\}=\int_{0}^{t-\frac{R^{*}}{c}} \int_{\Delta l_{j}} \int_{\Delta \Delta_{i}}\{f\}_{j}\{f\}_{i}^{T} H_{1} d x^{\prime} d x\{I(\tau)\}_{i} d \tau \\
& \left\{\hat{C}^{n}\right\}=\int_{0}^{t-\frac{R_{0}^{*}}{c}-\frac{2 n L}{c}-\frac{x}{c}} \int_{\Delta l_{j}} \int_{\Delta \Delta_{i}}\{f\}_{j}\{f\}_{i}^{T} H_{2} d x^{\prime} d x\{I(\tau)\}_{i} d \tau \\
& \left\{\hat{D}^{n}\right\}=\int_{0}^{t-\frac{R_{L}^{*}}{c}-\frac{(2 n+1) L}{c}-\frac{x}{c}} \int_{\Delta l_{j}} \int_{\Delta l_{i}}\{f\}_{j}\{f\}_{i}^{T} H_{3} d x^{\prime} d x\{I(\tau)\}_{i} d \tau
\end{aligned}
$$

where

$$
\begin{aligned}
& H_{1}=\frac{r^{\prime \prime}\left(\theta, t-\frac{R^{*}}{c}-\tau\right)}{4 \pi R^{*}} \\
& H_{2}=\frac{r^{\prime \prime}\left(\theta, t-\frac{R_{0}^{*}}{c}-\frac{2 n L}{c}-\frac{x}{c}-\tau\right)}{4 \pi R_{0}^{*}} \\
& H_{3}=\frac{r^{\prime \prime}\left(\theta, t-\frac{R_{L}^{*}}{c}-\frac{(2 n+1) L}{c}-\frac{x}{c}-\tau\right)}{4 \pi R_{0}^{*}}
\end{aligned}
$$

Assembling the local matrices and vectors into the global ones yields the global matrix system which can be written in the form:

$$
\left.[A]\{I\}\right|_{t-\frac{R}{c}}=\left.\{g\}\right|_{\substack{\text { previous time } \\ \text { instants }}}+\left.\{\hat{g}\}\right|_{\substack{\text { previous sime } \\ \text { instants }}}
$$

where

$$
\begin{aligned}
& \{g\}=\left.\left[A^{*}\right]\{I\}\right|_{t-\frac{R^{*}}{c}}+\left.[B]\{E\}\right|_{t-\frac{\left|x-x^{*}\right|}{c}} \\
& +\left.[C]\left\{\sum_{n=0}^{\infty} I^{n}\right\}\right|_{t-\frac{R_{0}}{c}-\frac{2 n L}{c}-\frac{x}{c}}-\left.\left[C^{*}\right]\left\{\sum_{n=0}^{\infty} I^{n}\right\}\right|_{t-\frac{R_{0}^{*}}{c}-\frac{2 n L}{c}-\frac{x}{c}}-\left.[B]\left\{\sum_{n=0}^{\infty} E^{n}\right\}\right|_{t-\frac{x^{\prime}}{c}-\frac{2 n L}{c}-\frac{x}{c}} \\
& -\left.[D]\left\{\sum_{n=0}^{\infty} I^{n}\right\}\right|_{t-\frac{R_{L}}{c}-\frac{(2 n+1) L}{c}-\frac{x}{c}}+\left.\left[D^{*}\right]\left\{\sum_{n=0}^{\infty} I^{n}\right\}\right|_{t-\frac{R_{L}^{*}-(2 n+1) L}{c}-\frac{x}{c}}+\left.[B]\left\{\sum_{n=0}^{\infty} E^{n}\right\}\right|_{t-\frac{L-x^{\prime}}{c}-\frac{(2 n+1) L}{c}-\frac{x}{c}} \\
& +\left.[D]\left\{\sum_{n=0}^{\infty} I^{n}\right\}\right|_{t-\frac{R_{L}}{c}-\frac{2 n L}{c}-\frac{L-x}{c}}-\left.\left[D^{*}\right]\left\{\sum_{n=0}^{\infty} I^{n}\right\}\right|_{t-\frac{R_{L}^{*}}{c}-\frac{2 n L}{c}-\frac{L-x}{c}}-\left.[B]\left\{\sum_{n=0}^{\infty} E^{n}\right\}\right|_{t-\frac{L-x^{\prime}}{c}-\frac{2 n L}{c}-\frac{L-x}{c}} \\
& -\left.[C]\left\{\sum_{n=0}^{\infty} I^{n}\right\}\right|_{t-\frac{R_{0}}{c}-\frac{(2 n+1) L}{c}-\frac{L-x}{c}}+\left.\left[C^{*}\right]\left\{\sum_{n=0}^{\infty} I^{n}\right\}\right|_{t-\frac{R_{0}^{*}}{c}-\frac{(2 n+1) L}{c}-\frac{L-x}{c}}+\left.[B]\left\{\sum_{n=0}^{\infty} E^{n}\right\}\right|_{t-\frac{x^{\prime}}{c}-\frac{(2 n+1) L}{c}-\frac{L-x}{c}}
\end{aligned}
$$

and

$$
\begin{aligned}
\{\hat{g}\}=\left.\{\hat{A}\}\right|_{t-\frac{R^{*}}{c}} & -\left.\left\{\sum_{n=0}^{\infty} \hat{C^{n}}\right\}\right|_{t-\frac{R_{0}^{*}}{c}-\frac{2 n L}{c}-\frac{x}{c}}+\left.\left\{\sum_{n=0}^{\infty} \hat{D}^{n}\right\}\right|_{t-\frac{R_{L}^{*}}{c}-\frac{(2 n+1) L}{c}-\frac{x}{c}} \\
& +\left.\left\{\sum_{n=0}^{\infty} \hat{D}^{n}\right\}\right|_{t-\frac{R_{L}^{*}}{c}-\frac{2 n L}{c}-\frac{L-x}{c}}-\left.\left\{\sum_{n=0}^{\infty} \hat{C}^{n}\right\}\right|_{t-\frac{R_{0}^{*}}{c}-\frac{(2 n+1) L}{c}-\frac{L-x}{c}}
\end{aligned}
$$


Applying the weighted residual approach in the time domain, and using the Dirac impulses as weight functions provides the time sampling, and the following recurrent formula is obtained:

$$
\left.I_{j}\right|_{t_{k}}=\frac{\left.\sum_{i=1}^{N^{s}} a_{j i} I_{j}\right|_{t_{k}-\frac{R}{c}}-\left.g_{j}\right|_{\begin{array}{l}
\text { previous time } \\
\text { instants }
\end{array}}-\left.\hat{g}_{j}\right|_{\begin{array}{c}
\text { previous time } \\
\text { instants }
\end{array}}}{a_{j j}}
$$

where $\left.I_{j}\right|_{t_{k}}$ is current for the $j$-th space node at $k$-th time instant, $N$ is total number of space segments, while the overbar indicates the absence of diagonal members.

It is worth noting that the numerical calculation of convolution integrals is rather tedious task leading to tremendously large computational time of the overall method. The main advantage of the method, on the other hand, is its unconditional stability.

\subsection{Numerical results for an overhead wire}

Computational example is related to a transient scattering from a straight thin wire of length is $L=1 \mathrm{~m}$, radius $a=2 \mathrm{~mm}$, located at height $h=0.25 \mathrm{~m}$ above ground with permittivity $\varepsilon_{\mathrm{r}}=10$, while the conductivity is varied. The wire is illuminated by the tangential electromagnetic pulse (EMP) plane wave:

$$
E_{x}^{i n c}(t)=E_{0}\left(e^{-a t}-e^{-b t}\right), \quad t \geq 0
$$

with: $E_{0}=1 \mathrm{~V} / \mathrm{m}, a=4 \cdot 10^{7} \mathrm{~s}^{-1}, b=6 \cdot 10^{8} \mathrm{~s}^{-1}$.

Fig. 2 shows the transient current induced at the wire center for different ground conductivities.

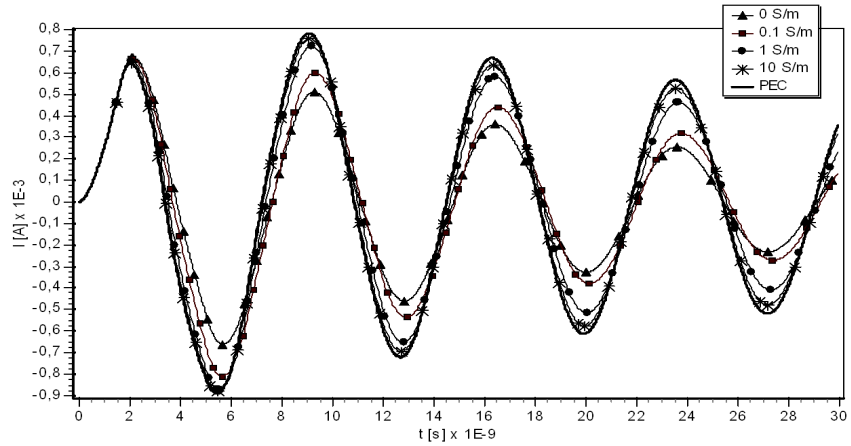

Figure 2: $\quad$ Transient current at the wire center, $L=1 \mathrm{~m}, a=2 \mathrm{~mm}, h=0.25, \varepsilon_{\mathrm{r}}=10$.

The influence of the ground conductivity to the transient response is particularly visible from around $0.1 \mathrm{~S} / \mathrm{m}$ to $1 \mathrm{~S} / \mathrm{m}$. 


\section{Pocklington integral equation formulation}

The geometry of interest, shown in Fig. 3, is the horizontal grounding electrode of length $L$ and radius $a$, buried in a lossy medium at depth $d$ and excited at one end by an equivalent current source.

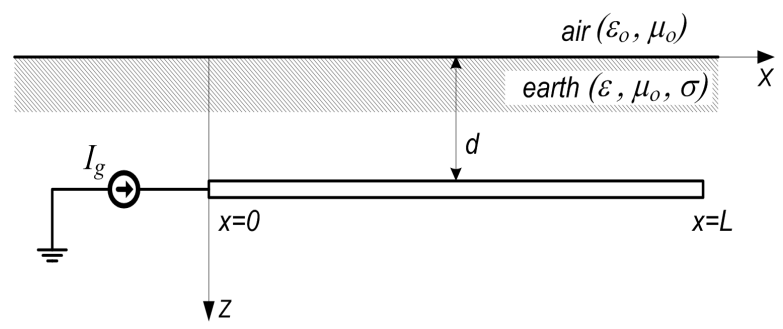

Figure 3: Horizontal grounding wire energized by a current generator Ig.

Since the electric field excitation along the electrode does not exist i.e.:

$$
E_{x}^{e x c}=0
$$

assuming the certain set of approximation [7] the transient current induced along the electrode is governed by the following form of the Pocklington equation:

$$
\begin{aligned}
& {\left[-v^{2} \frac{\partial^{2}}{\partial x^{2}}+\frac{\partial^{2}}{\partial t^{2}}+\frac{\sigma}{\varepsilon} \frac{\partial}{\partial t}\right] \cdot} \\
& {\left[\frac{\mu}{4 \pi} \int_{0}^{L} I\left(x^{\prime}, t-R / v\right) \frac{e^{-\frac{t}{\tau_{g}} \frac{R}{v}}}{R} d x^{\prime}-\int_{-\infty}^{t} \int_{0}^{L} \Gamma_{r e f}(\theta, \tau) \frac{I\left(x^{\prime}, t-R^{*} / v-\tau\right)}{4 \pi R^{*}} \frac{e^{-\frac{t}{\tau_{g}} \frac{R^{*}}{v}}}{R^{*}} d x^{\prime} d \tau\right]=0}
\end{aligned}
$$

where the reflection coefficient is given by [7]:

$$
\Gamma_{r e f}(t)=-\left[\frac{\tau_{1}}{\tau_{2}} \delta(t)+\frac{1}{\tau_{2}}\left(1-\frac{\tau_{1}}{\tau_{2}}\right) e^{-t / \tau_{2}}\right]
$$

where $\tau_{1}$ and $\tau_{2}$ are the time constants characterized by a lossy medium [7]:

$$
\tau_{1}=\frac{\varepsilon_{r}-1}{\sigma} \varepsilon_{0}, \quad \tau_{2}=\frac{\varepsilon_{r}+1}{\sigma} \varepsilon_{0}
$$

Note that the current source is included into the integral equation scheme trough the boundary condition: 
140 Boundary Elements and Other Mesh Reduction Methods XXXIII

$$
I(0)=I_{g}
$$

which is inserted subsequently in the global matrix system [2].

\subsection{BEM procedure for Pocklington equation}

It should be emphasized that, for the sake of simplicity, this paper considers only the case of an infinite lossy medium. Applying the weighted residual approach and performing space-discretization i.e. in matrix form one obtains the following time domain differential equation:

$$
[M] \frac{\partial^{2}}{\partial t^{2}}\left\{I\left(t^{\prime}\right)\right\}+[C] \frac{\partial}{\partial t}\left\{I\left(t^{\prime}\right)\right\}+[K]\left\{I\left(t^{\prime}\right)\right\}=0
$$

where the space dependent matrices are given by:

$$
\begin{aligned}
M_{j i} & =\frac{1}{v^{2}} \int_{\Delta l_{j}} \int_{\Delta l_{i}}\{f\}_{j}\{f\}_{i}^{T} \frac{e^{-\frac{T}{\tau}}}{R} d x^{\prime} d x \\
C_{j i} & =\frac{\sigma}{\varepsilon} \int_{\Delta l_{j}} \int_{\Delta l_{i}}\{f\}_{j}\{f\}_{i}^{T} \frac{e^{-\frac{T}{\tau}}}{R} d x^{\prime} d x \\
K_{j i} & =\frac{\mu}{4 \pi} \int_{\Delta l_{j}} \int_{\Delta l_{i}}\{D\}_{j}\{D\}_{i}^{T} \frac{e^{-\frac{T}{\tau}}}{R} d x^{\prime} d x
\end{aligned}
$$

where $\{\mathrm{D}\}$ stands for the shape functions derivatives and: $\tau=\frac{2 \varepsilon}{\sigma}$ and $T=\frac{R}{v}$. Finally, differential equation (21) is solved performing the marching-on-in-time procedure presented in [5]:

$$
\begin{aligned}
\sum_{i=1}^{n}\left[M_{j i}+\beta \Delta t^{2} K_{j i}\right] I_{i}^{k} & =-\sum_{i=1}^{n}\left[-2 M_{j i}+\left(\frac{1}{2}-2 \beta+\gamma\right) \Delta t^{2} K_{j i}\right] I_{i}^{k-1} \\
& -\sum_{n=1}^{n}\left[M_{j i}+\left(\frac{1}{2}+\beta-\gamma\right) \Delta t^{2} K_{j i}\right] I_{i}^{k-2}
\end{aligned}
$$

where $\Delta t$ stands for the time increment and the stability of the procedure is achieved by choosing [5] $\gamma=1 / 2$ and $\beta=1 / 4$.

\subsection{Numerical results for grounding electrode}

Computational example is related to the grounding electrode of length $L=10 \mathrm{~m}$, radius $a=5 \mathrm{~mm}$, immersed in the lossy ground with $\varepsilon_{\mathrm{r}}=10$, and $\sigma=0.001 \mathrm{~S} / \mathrm{m}$. The electrode is excited with the double exponential current pulse: 


$$
i(t)=I_{0} \cdot\left(e^{-a t}-e^{-b t}\right), \quad t \geq 0
$$

defined with $I_{0}=1.1043 \mathrm{~A}, \mathrm{a}=0.07924 \cdot 10^{7} \mathrm{~s}^{-1}, \mathrm{~b}=4.0011 \cdot 10^{7} \mathrm{~s}^{-1}$.

The transient current induced at the centre of the electrode obtained via the presented direct time domain approach and the indirect frequency domain approach GB-IBEM with Fast Fourier Transform (FFT) is shown in Fig. 4.

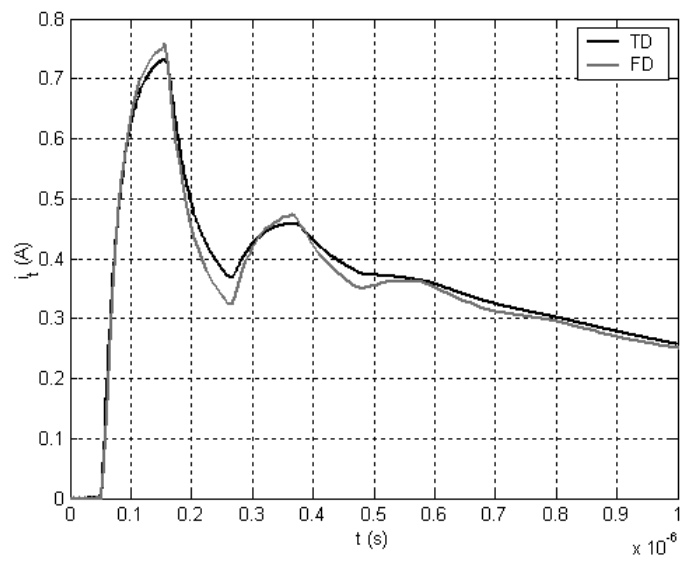

Figure 4: $\quad$ Transient current induced at the centre of the wire.

An acceptable agreement between the results computed via different approaches can be observed.

\section{Concluding remarks}

The paper deals with the transient analysis of thin wire structures in the presence of a lossy half-space, based on the time domain Hallen and Pocklington integral equation, respectively. The finite conductivity of the ground is taken into account via the corresponding reflection coefficients. The both types of integral equations are handled via the certain scheme of Galerkin-Bubnov Indirect Boundary Element Method (GB-IBEM) The strength and weaknesses of both approaches are emphasized and some illustrative examples related to overhead wires and grounding electrodes are presented within this work.

\section{References}

[1] F. M. Tesche, M. Ianoz, and T. Karlsson, EMC Analysis methods and computational models. New York: Wiley Interscience, 1997.

[2] D. Poljak, EMC Advanced Modelling in Computational electromagnetic Compatibility, John Wiley and Sons, New York 2007. 
[3] E.K. Miller, J.A. Landt: "Direct time-domain techniques for transient radiation and scattering from wires", IEEE Trans.AP, Vol. 68, No.11, pp. 1396-1424, Nov. 1980.

[4] D. Poljak, C.Y. Tham, A. McCowen: "Transient Response of Nonlinearly Loaded Wires in a Two Media Configuration", IEEE Trans.EMC, Vol. 46, No.1, pp. 121-125, 2004.

[5] V. Doric, D. Poljak, V. Roje, Direct Time Domain Analysis of a Lightning Rod Based on the Antenna Theory, EMC Symposium, 2009.

[6] D. Poljak, Transient Response of Straight Thin Wires Located at Different Heights Above a Ground Plane Using Antenna Theory and Transmission Line Approach, IEEE Trans.EMC, Vol.52, No.1, pp. 108-1116, 2010.

[7] D. Poljak, EABE 2008, Time domain modeling of a thin wire in a twomedia configuration featuring a simplified reflection/transmission coefficient approach, Engineering Analysis with Boundary Elements, EABE 33, pp. 283-293., 2009.

[8] S. Antonijevic, D. Poljak, On Time Domain Numerical Modeling of a Thin Wire Above a Lossy Ground, SoftCOM 2010, Split, Brac Island, Croatia, Sept. 2010. 